\title{
Abnormal Causal Connectivity of Left Superior Temporal Gyrus in Drug-naïve First- episode Adolescent-onset Schizophrenia: A Resting-state fMRI Study
}
H. Lyu ${ }^{1}$, X. Wang ${ }^{2}$,
B. Sun ${ }^{3}$, Z. Zhao 4
D. Shang ${ }^{2}$, F. Pan ${ }^{1}$, Y. Xu1,
D. $\mathrm{Xu}^{5}$, M. Huang ${ }^{1}$.

1. The First Affiliated Hospital- Zhejiang University School of Medicine, Department of Psychiatry, Hangzhou, China.

2. Zhejiang University, School of Medicine, Hangzhou, China.

3. Ningbo Mental Hospital, Psychiatry, Ningbo, China.

4. East China Normal University, Shanghai Key Laboratory of Magnetic Resonance, Shanghai, China.

5. Columbia University, New York State Psychiatric Institute, New York, USA.

Background
Functional brain abnormalities have been studied in AOS
cases. However, the alterations of causal connectivity
between brain regions in AOS remains unclear.

\section{Method}

Thirty-two first-episode drug-naïve AOS patients and 27 healthy controls $(\mathrm{HC})$ were recruited for resting-state functional MRI scanning. The brain region with the betweengroup difference in Regional homogeneity ( $\mathrm{ReHo}$ ) values was chosen as a seed to perform the Granger causality analysis (GCA) and further detect the alterations of causal connectivity in AOS. Both receiver-operating characteristic analysis and probabilistic neural network were applied to test the possibility of using abnormal brain connectivity for distinguishing cases from controls.

Tablel Brain regions showing significant effective connections difference both to and from left STG between $\mathrm{AOS}$ patients and $\mathrm{HC}$.

\begin{tabular}{|c|c|c|c|c|c|c|c|}
\hline \multirow[b]{2}{*}{ Brain regions } & \multirow[t]{2}{*}{ Side } & \multirow[t]{2}{*}{ Brodmann Area } & \multicolumn{3}{|c|}{ MNI coordinates } & \multirow{2}{*}{$\begin{array}{l}\text { Peak } \\
\text { T-value }\end{array}$} & \multirow{2}{*}{$\begin{array}{l}\text { Cluster } \\
\text { size }\end{array}$} \\
\hline & & & $x$ & Y & $z$ & & \\
\hline \multicolumn{8}{|l|}{ Left STG $\rightarrow$ other regions } \\
\hline Superior frontal gyrus & $R$ & $B A 8 / 9$ & 15 & 27 & 33 & -3.77 & 91 \\
\hline Angular gyrus & $R$ & BA39 & 39 & -75 & 36 & -3.51 & 99 \\
\hline Insula & $R$ & BA13 & 48 & -12 & 12 & 4.87 & 97 \\
\hline \multicolumn{8}{|l|}{ Other regions $\rightarrow$ left STG } \\
\hline Middle occipital gyrus & $R$ & BA17/18 & 9 & -87 & -3 & 5.03 & 125 \\
\hline
\end{tabular}

STG, Superior Temporal Gyrus; MNI, Montreal Neurological Institute; AOS, adolescent-onset schizophrenia; HC, healthy control

Figure 1. Brain regions showing effective connectivity alterations in AOS patients compared with $\mathrm{HC}$

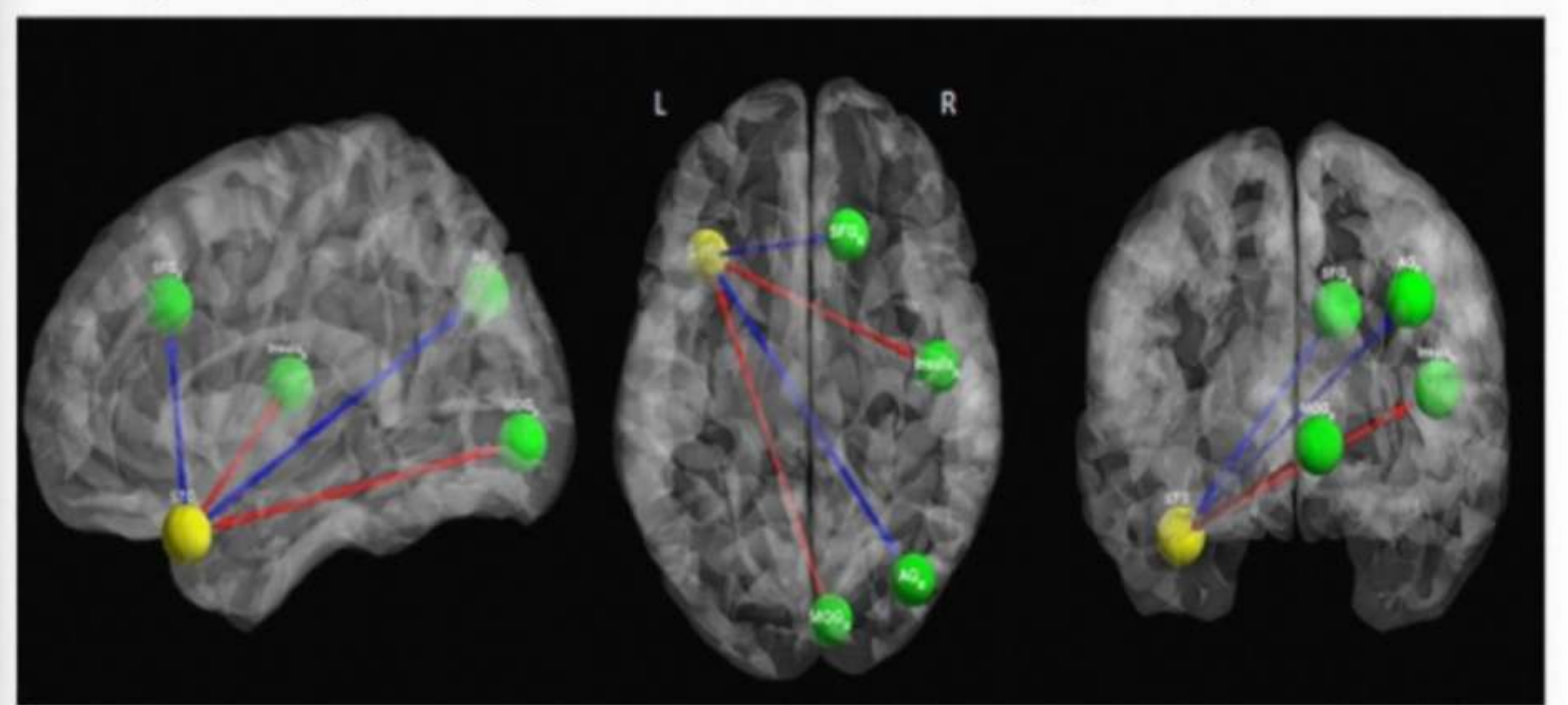

AOS, Adolescent onset schizophrenia; $\mathrm{HC}$, healthy control.

Significant lower outgoing Granger causality (GC) values from left superior temporal gyrus (STG) to right superior frontal gynus (SFG) and right angular gynus (Ag) were detected in GC mapping for AOS (corrected $\mathrm{p}<0.05$ ). Significant stronger causal outflow from left STG to right insula and stronger causal inflow from right middle occipital gyrus (MOG) to left STG were also observed in AOS patients (corrected $p<0.05$ ).

\section{Results}

AOS patients exhibited increased ReHo values in left superior temporal gyrus (STG) compared with HC. Significantly decreased values of outgoing Granger causality from left STG to right superior frontal gyrus and right angular gyrus were observed in GC mapping for AOS. Significantly stronger causal outflow from left STG to right insula and stronger causal inflow from right middle occipital gyrus (MOG) to left STG were also observed in AOS patients. Based on assessments of the two strengthened causal connectivity of the left STG with insula and MOG, a cross-validated probabilistic neural network model could discriminate all patients from controls with remarkable accuracy at $94.9 \%$.

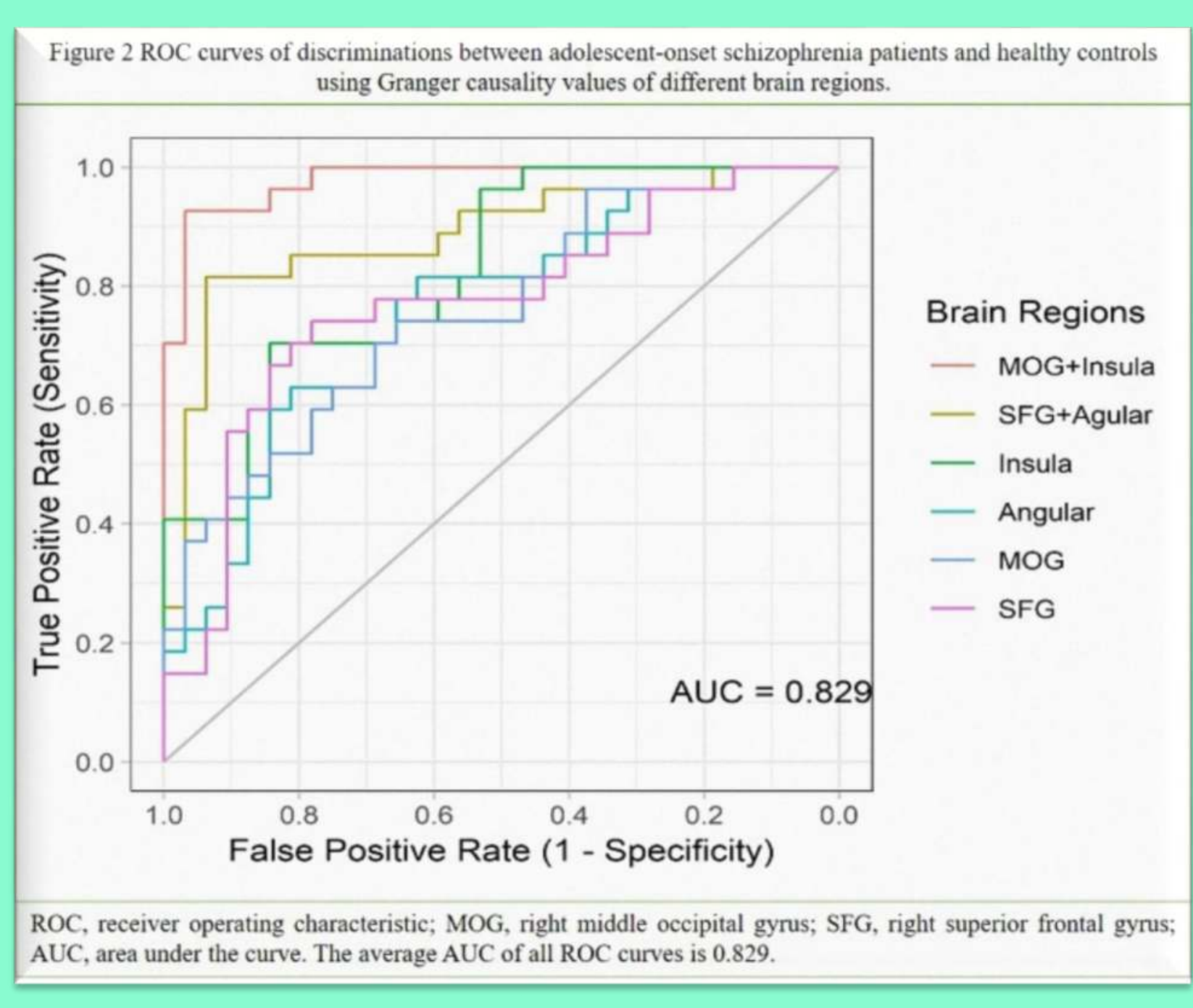

\section{Conclusions}

Patients with drug-naïve first-episode AOS exhibit brain functional connectivity abnormalities in left STG. The alterations of directional connections in left STG may play an important role in the pathogenesis of AOS and serve as potential biomarkers for the disease.

Presented at EPA 2019

$27^{\text {th }}$ European Congress of Psychiatry Warsaw, Poland

6-9 April 2019 